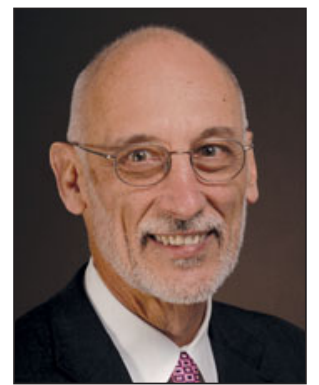

Robert Nemanich

Guest Editor for this issue of MRS Bulletin

Department of Physics, Arizona State University, USA; email robert.nemanich@asu.edu.

Nemanich is a professor of physics at Arizona State University. He completed his BS and MS degrees in physics at Northern Illinois University and his $\mathrm{PhD}$ degree at the University of Chicago. He then joined Xerox PARC and later moved to North Carolina State University. He is a Fellow of MRS and was MRS President in 1998. He is also a Fellow of the American Physical Society and was the 2011 Chair of the Division of Materials Physics. He served as editor-in-chief of Diamond and Related Materials (1999-2012) and has mentored over 70 graduate students. His areas of interest include the growth, processing, and interfaces of electronic materials.

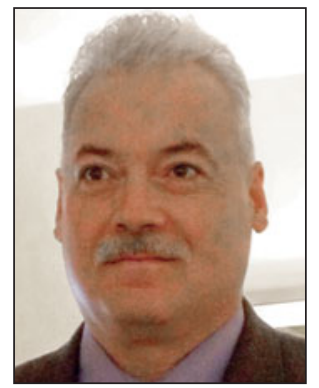

\section{John Carlisle}

Guest Editor for this issue of MRS Bulletin

Advanced Diamond Technologies, Inc., IL, USA; email carlisle@thindiamond.com.

Carlisle is the founder, Chief Technical Officer, and GM of the Environmental Products Business Unit of Advanced Diamond Technologies. He received his BS degree in physics and mathematics from Texas A\&M University-Commerce (1986) and his PhD degree in physics from the University of Illinois at Urbana-Champaign (1993). From 1993 to 1996, he was a postdoctoral research associate at Lawrence Livermore National Laboratory. He then was an assistant professor of physics at Virginia Commonwealth University until 2000, when he became a physicist at Argonne National Laboratory until 2006. He founded ADT in 2003. His work is on nanostructured carbon materials, focusing on the synthesis and applications of ultrananocrystalline diamond thin films. He is a four-time R\&D 100 Award winner, and ADT was named a Technology Pioneer in 2007 by the World Economic Forum. He has authored approximately 130 publications.

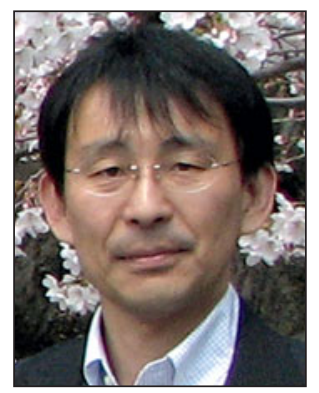

\section{Atsushi Hirata}

Guest Editor for this issue of MRS Bulletin

Department of Mechanical Sciences and Engineering, Tokyo Institute of Technology, Japan; tel. 81-3-5734-2163; and email ahirata@ctrl.titech.ac.jp.

Hirata is an associate professor at Tokyo Institute of Technology, where he received his BS, MS, and Dr. Eng. degrees in mechanical engineering. His interests include surface engineering and materials processing technology, including thinfilm coating, solid lubrication, wear monitoring, polishing, high energy beam processing, sintering, and diffusion bonding. His research is on carbon materials such as CVD diamond, detonation nanodiamond, amorphous carbon coating, carbon nanotube, and carbon onion.

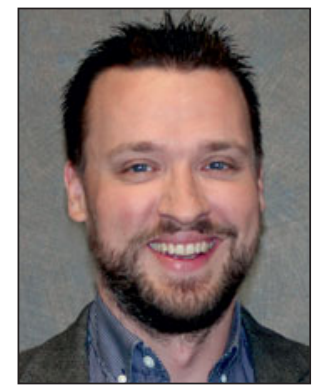

Ken Haenen

Guest Editor for this issue of MRS Bulletin

Institute for Materials Research and IMOMEC Hasselt University and IMEC vZW,

Diepenbeek, Belgium; tel. +3211268 875; and email ken.haenen@uhasselt.be.

Haenen obtained his MS (1997) and PhD (2002) degrees, both in physics, from KU Leuven and Hasselt University. After a postdoc period at the National Institute for Materials Science (NIMS, formerly NIRIM), he became group leader of wide bandgap materials at the Institute for Materials Research at Hasselt University (2004), then became an appointed professor of experimental physics (2008). His research is on CVD diamond, including combinations with AIN, BN, and small molecules. $\mathrm{He}$ is serving as a 2015 MRS Spring Meeting Chair and chairs/organizes the DCM, NDNC, and SBDD diamond and carbon materials conferences. He is editor of Diamond and Related Materials and annual guest editor of Physica Status Solidi $A$ for related special issues.

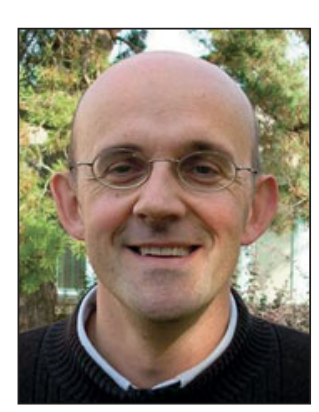

Jean-Charles Arnault

CEA LIST Atomic Energy and Alternative

Energies Commission, France;

email jean-charles.arnault@cea.fr.

Arnault is a research director at the Atomic Energy and Alternative Energies Commission (CEA). After obtaining his PhD degree in 1993, he was an assistant professor at Strasbourg University until 2007, when he joined the Diamond Sensors Laboratory at CEA. His expertise concerns diamond nucleation and growth, interactions between MPCVD plasmas with surfaces using in situ surface analysis methods, and electron microscopies. Since 2008, his research has focused on surface modifications of diamond nanoparticles using plasma or thermal treatments to control their surface terminations and confer new surface properties.

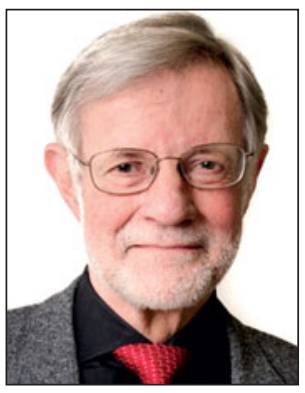

Jes Asmussen

Michigan State University, USA; email asmussen@egr.msu.edu.

Asmussen is a university distinguished professor and the Richard M. Hong Chaired Professor in the Department of Electrical and Computer Engineering at Michigan State University. His research is on the development and application of microwave discharges. His patented technology has resulted in numerous microwave plasma and material processing machines sold throughout the United States, Europe, and Asia.

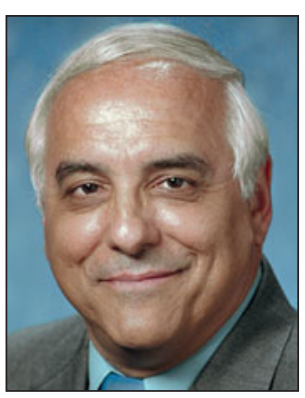

\section{Orlando Auciello}

University of Texas at Dallas, USA;

tel. 972-883-4731; and

email orlando.auciello@utdallas.edu.

Auciello is an endowed chair professor at the University of Texas at Dallas. He holds MS and $\mathrm{PhD}$ degrees in physics from Balseiro Institute University of Cuyo, Argentina. He works on multifunctional oxide and novel ultrananocrystalline diamond films and application to devices commercialized by two companies he co-founded (Advanced Diamond Technologies and Original Biomedical Implants). He has published 20 books and 500 articles, holds 17 patents, and has organized/lectured at many conferences. He is associate editor of Applied Physics Letters. He was a MRS board member and MRS President in 2013. His awards include seven R\&D 100 Awards, Hispanic Achievement, National Laboratories, and AAAS/ MRS Fellowships. 


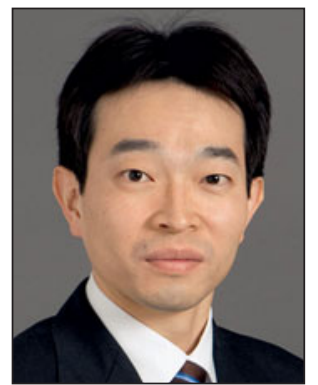

Yasuaki Einaga

Department of Chemistry, Keio University, Japan; tel. 81-45-566-1704; and email einaga@chem.keio.ac.jp.

Einaga is a professor in the Department of Chemistry at Keio University. He received his BS (1994), MS (1996), and PhD (1999) degrees from the University of Tokyo, where he worked for two years as a research associate. He became an assistant professor at Keio University (2001), where he was promoted to associate professor (2003) then to professor (2011). He is a director of a Japan Science and Technology Agency CREST project from 2011. His research includes functional materials science, photochemistry, electrochemistry, and diamond electrodes.

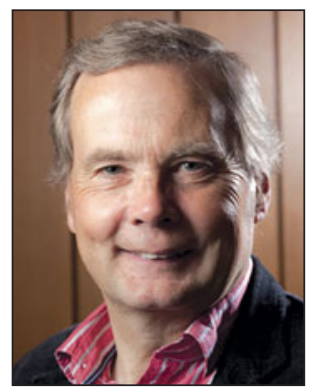

\section{John Foord}

Department of Chemistry, Chemistry Research Laboratory, Oxford University, UK;

tel. +44 1865 276967; and

email john.foord@chem.ox.ac.uk.

Foord is a professor of chemistry at Oxford University. He graduated with a bachelor's degree in natural sciences and a PhD degree from the University of Cambridge. After a lectureship at the University of Southampton, he moved to his present position. His research is on diamond thin films, electrochemistry, fuel cells, and polymer nanocomposites.

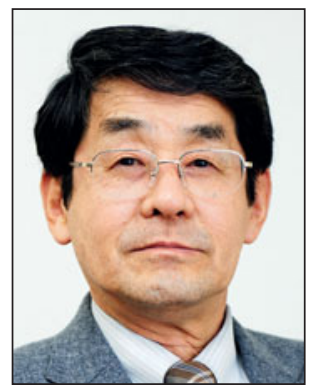

Naoji Fujimori

EDP Corporation, Japan;

email n.fujimori@d-edp.jp.

Fujimori is the president of EDP Corporation. He received his master's degree in metallurgy (1975) from the University of Tokyo and studied diamond semiconductors in his doctoral work in electronic engineering (1995) at Kyoto University. In 1975, he joined Sumitomo Electric Industries, Ltd. where he started CVD diamond growth technology. In 2003, he joined the National Institute of Advanced Industrial Science and Technology (AIST) as the director of the Diamond Research Center. In 2009, he established EDP Co. manufacturing single-crystal diamonds.

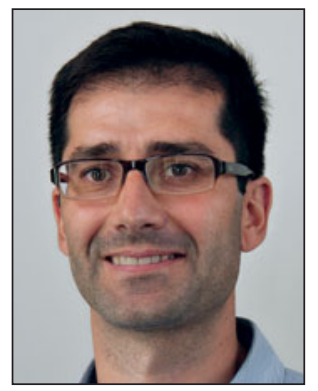

\section{Jose Garrido}

Walter Schottky Institute and Physics

Department, Technische Universität München, Germany; tel. +49 89289 12766; and email garrido@wsi.tum.de.

Garrido has been a research associate at the Walter Schottky Institute since 2000 and a senior lecturer in the Physics Department of the Technische Universität München since 2011. He received his $\mathrm{PhD}$ degree from the Universidad Politécnica de Madrid in 2000. His research focuses on the use of carbon materials such as diamond, graphene, and organic semiconductors for applications in electronics, bioelectronics, and biosensing.

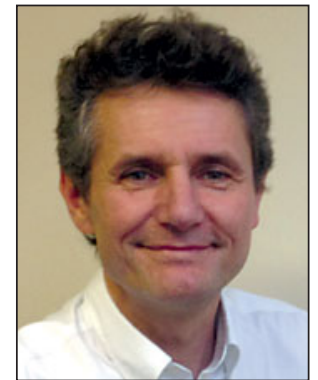

Etienne Gheeraert

University of Grenoble Alpes, Institut NEEL,

France; Etienne.Gheeraert@neel.cnrs.fr.

Gheeraert is a professor at the Université of Grenoble Alpes and is in charge of the wide bandgap semiconductor group at Institut Néel (CNRS and Université Joseph Fourier). After graduation from the École Normale Supérieure de Cachan in electrical engineering, he completed a PhD degree in physics at the Université Joseph Fourier, where he joined as an assistant professor (1992) followed by professor (2004). From 1998 to 1999, he worked at NIMS and was attaché for science and technology at the Embassy of France in Japan from April 2006-September 2008.

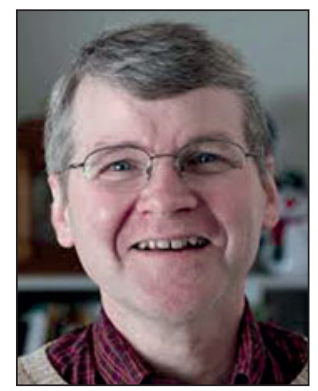

Robert J. Hamers

Department of Chemistry, University

of Wisconsin-Madison, USA

email rjhamers@wisc.edu.

Hamers is a professor of chemistry and the director of the Center for Sustainable Nanotechnology at the University of Wisconsin-Madison. He received his $\mathrm{PhD}$ degree from Cornell University (1986). From 1985-1990, he was a visiting scientist followed by a research staff member at the IBM T.J. Watson Research Center. He joined the University of WisconsinMadison as an associate professor in 1990 and was promoted to full professor in 1994. His research involves surface and interfacial phenomena, specifically applications in renewable energy and in environmental science.

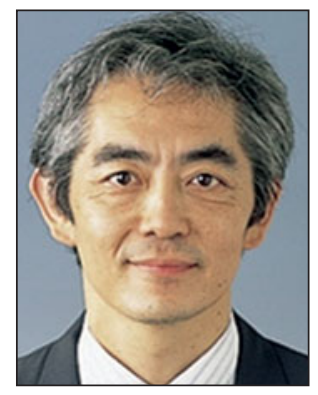

Hiroshi Kawarada

Department of Electronics and Phonics

Systems and Department of Nanoscience and Nanotechnology, Waseda University, Japan; tel. +81352863391 ; and email kawarada@waseda.jp.

Kawarada is a professor in the Department of Electronics and Phonics Systems and the Department of Nanoscience and Nanotechnology at Waseda University, where he also received his BS, ME, and Dr. Eng. (1985) degrees. He was an assistant professor at Osaka University (1986-1990) before joining Waseda University in 1990. His research focuses on surface functionalization of diamond for transistors for high-frequency operation, quantum effect, biosensing, and high voltage application; and nano-, bio-, and power electronics using carbon-based materials, including diamond.

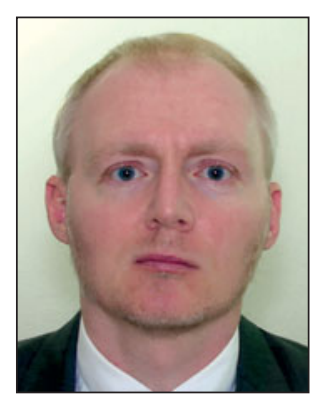

\section{Franz A. Koeck}

Department of Physics, Arizona State University, USA; email franz.koeck@asu.edu.

Koeck is a research physicist in the Physics Department at Arizona State University (ASU). He received his master's degree in physics at North Carolina State University working on plasma-assisted chemical vapor deposition of carbon thin films, electron emission, and directenergy conversion from carbon-based materials. At ASU, he researches direct-energy conversion from doped diamond films with a focus on surface ionization effects. 


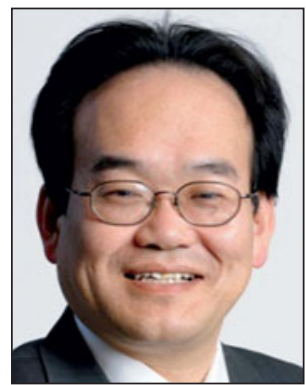

Yasuo Koide

National Institute for Materials Science,

Japan; email koide.yasuo@nims.go.jp.

Koide is a group leader of a wide bandgap materials group, a platform director of the Nanofabrication Platform, and a deputy managing director of the Center of Materials Research for Low Carbon Emission at the National Institute for Materials Science, where he has been since 2002. He received his PhD degree from Nagoya University in 1988, where he was an assistant professor, followed by an associate professor at Kyoto University prior to his present position. He has more than 300 publications.

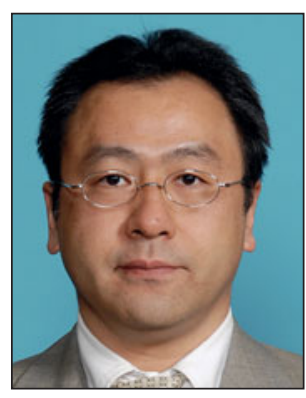

Satoshi Koizumi

National Institute for Materials Science, Japan; email koizumi.satoshi@nims.go.jp. Koizumi is a principal researcher at the National Institute for Materials Science. He received his doctorate in electrical engineering at Aoyama Gakuin University and joined the Diamond Research Group at the National Institute for Research in Inorganic Materials in 1994. His research focuses on semiconducting diamond growth and device applications. He established the $n$-type diamond growth technique and succeeded in deep ultraviolet light-emitting diode formation by diamond $p-n$ junctions for the first time in 2001 .

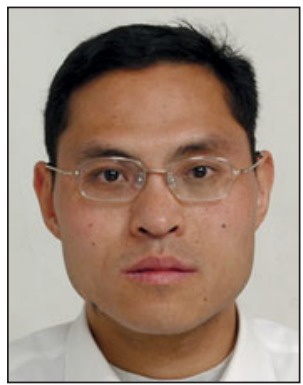

\section{Meiyong Liao}

National Institute for Materials Science,

Japan; tel. +81(29) 860-4508; and

email meiyong.liao@nims.go.jp.

Liao has been a senior researcher at the National Institute for Materials Science (NIMS) since 2008. He received his bachelor's (1996) and master's (1999) degrees from Lanzhou University and his PhD (2002) degree from the Institute of Semiconductors, Chinese Academy of Sciences. From 2002 to 2004, he was a visiting associate professor at Kyoto University before joining NIMS as a postdoctoral researcher in 2004. His research focuses on semiconductor electronic, photonic, and MEMS/NEMS devices.

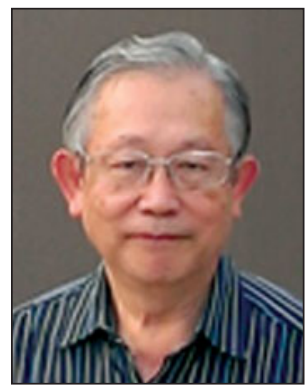

\section{I-Nan Lin}

Department of Physics, Tamkang University,

Taiwan; tel. +886-2-26268907; and

email inanlin@mail.tku.edu.tw.

Lin is a senior professor at Tamkang University. He received his bachelor's degree in physics from National Taiwan Normal University, his MS degree from National Tsing-Hua University, and his $\mathrm{PhD}$ degree in materials science from the University of California, Berkeley (1979). He was a senior researcher in the Materials Science Center at Tsing-Hua University prior to his present position. His research focuses on high conductivity diamond films and their applications and TEM microscopic investigation of the microstructure evolution of diamond films in the microwave plasma-enhanced chemical vapor deposition process.

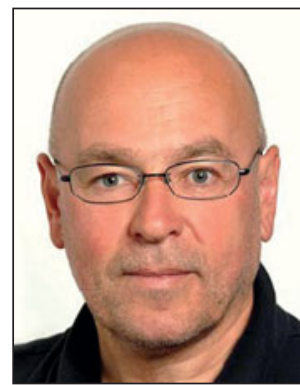

\section{Christoph Nebel}

Fraunhofer-Institute for Applied Solid

State Physics, Freiburg, Germany;

email christoph.nebel@iaf.fraunhofer.de.

Nebel is the head of the Micro- and NanoSensors Department of the Fraunhofer-Institute for Applied Solid State Physics, Freiburg, Germany. After graduating with a degree in electrical engineering at the University of Stuttgart in 1989 he was a postdoc at the Xerox Research Center (1990-1992). In 1993, he joined the Walter Schottky Institute at Technische Universität München, where he habilitated in 1998. He became team leader of the Biofunctionalized Device Team at the National Institute of Advanced Industrial Science and Technology (AIST), Diamond Research Center, Tsukuba, Japan, in 2004. In 2008, he joined the Fraunhofer Institute for Applied Solid State Physics.

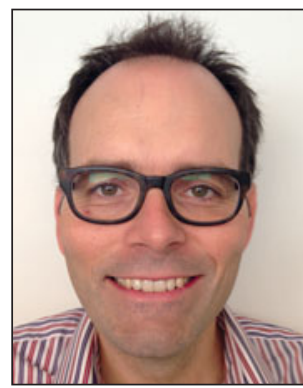

\section{Chris Pakes}

Department of Physics, La Trobe University, Victoria, Australia; tel. +61 39479 1485;

and email c.pakes@latrobe.edu.au.

Pakes is an associate professor and associate dean (research) at La Trobe University. He received his $\mathrm{PhD}$ degree from the University of Birmingham in 1999. He worked at the National Physical Laboratory and the University of Melbourne before joining the faculty at La Trobe University in 2007. His research focuses on molecular surface transfer doping of technologically interesting materials studied with a combination of UHV scanning probe microscopy, synchrotron-based photoemission, and low-temperature transport.

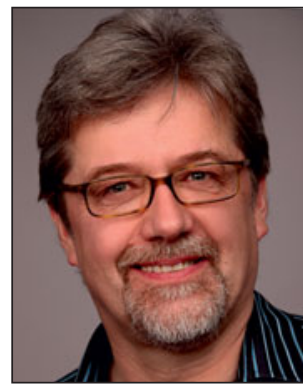

\section{Matthias Schreck}

Institute of Physics, University of Augsburg, Germany; email matthias.schreck@ physik.uni-augsburg.de.

Schreck is a research group leader at the Institute of Physics at the University of Augsburg. He received his diploma degree in physics from the University of Stuttgart (1987) and studied Langmuir-Blodgett films for his PhD degree in physical chemistry at the University of Tübingen (1990). In 1991, he joined the University of Augsburg, where he started chemical vapor deposition diamond activities. His research interests include epitaxial growth and structural analysis of oxide, metal, and diamond films; studies on diamond growth and nucleation; synthesis of single and mosaic crystals; and doping.

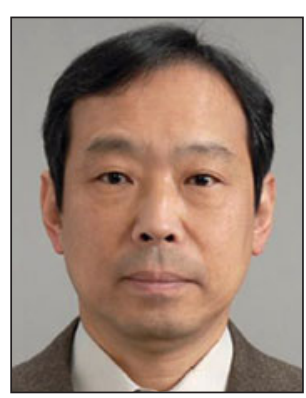

\section{Shinichi Shikata}

National Institute of Advanced Industrial

Science and Technology, Japan;

email s-shikata@aist.go.jp.

Shikata is principal research manager and GL of a diamond group at the National Institute of Advanced Industrial Science and Technology (AIST), where he has been since 2004. Since 2011, he concurrently serves the graduate school of Chiba University as a visiting professor. He received his BS and MS degrees from Kyoto University and his PhD degree from Osaka University. He joined Sumitomo Electric Industries Ltd. in R\&D of GaAs electron devices and diamond SAW devices for optical and wireless communication systems before joining AIST. He is a senior member of IEEE and a member of the JSAP and IEICE. His research interests include how diamond relates to power switching devices and wafers. 


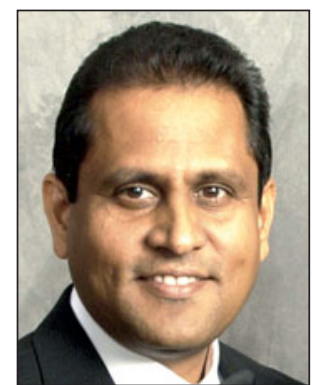

Anirudha Sumant

Argonne National Laboratory, IL, USA

tel.630-252-4854; and email sumant@anl.gov. Sumant is a materials scientist at the Center for Nanoscale Materials at Argonne National Laboratory. He received his $\mathrm{PhD}$ degree in electronic science from the University of Pune in 1998. His research interests include electronic and mechanical properties of carbon-based materials, surface chemistry, micro-/nanoscale tribology, and micro-nanofabrication. He has more than 80 publications and two book chapters, is the recipient of three R\&D 100 Awards, holds five patents granted (two licensed) with seven pending, and has given numerous invited talks. He is a member of MRS and AVS.

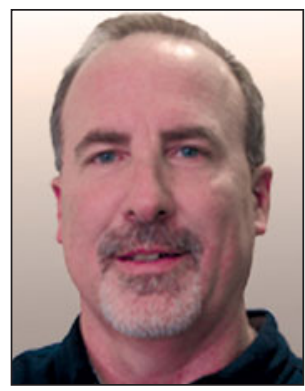

Greg Swain

Department of Chemistry, Michigan State University, USA; tel. 517-355-9715 x229;

and email swain@chemistry.msu.edu.

Swain is a professor and a member of the neuroscience program in the Department of Chemistry at Michigan State University. He received his BA degree from the University of Texas at Dallas (1987) and his PhD degree from the University of Kansas (1991). He was a JSPS/ NSF postdoctoral fellow at Tohoku University (1992-1993) and then joined the faculty in the Department of Chemistry and Biochemistry at Utah State University before his present position. His research interests include diamond materials science, physical and analytical electrochemistry, spectroelectrochemistry, and neurochemistry.
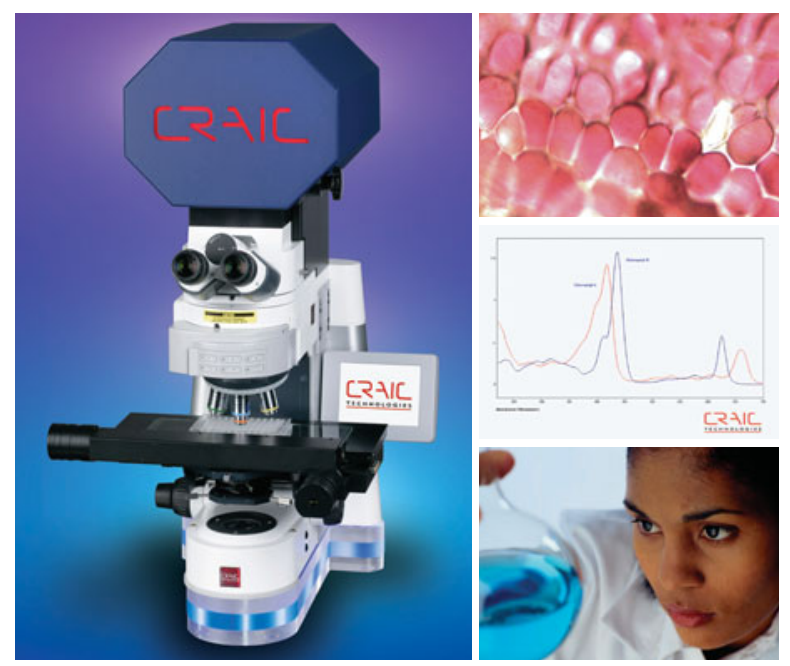

\section{SPECTROSCOPY oF MICROSCOPIC SAMPLES}

CRAIC Technologies UV-visible-NIR microscopes and microspectrophotometers are used for imaging and spectral analysis of sub-micron sized features with absorbance, reflectance, fluorescence, emission and polarized illumination. Capabilities include film thickness measurements, colorimetry and high resolution imaging in the UV, visible and NIR regions. Rapid \& accurate spectra \& images of microscopic samples: The Perfect Vision for Science ${ }^{\mathrm{TM}}$.

\section{MRS Booth 1023}

For more information, call 877.UV.CRAIC or visit our website at www.microspectra.com ○2011 CRAIC Technologies, Inc. San Dimas, California (USA).

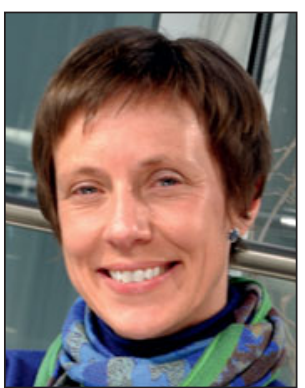

Sabine Szunerits

Interdisciplinary Research Institute, Parc de la Haute Borne, France; tel. +33 3625317 25; and email sabine.szunerits@iri.univ-lille1.fr. Szunerits has been a professor in chemistry since 2009 at the University Lille 1 attached to the Interdisciplinary Research Institute. She holds a PhD degree in organic electrochemistry from the Queen Mary and Westfield College and an MA degree in social science from the Open University of London. She became a member of the Institut Universitaire de France in 2010. She researches novel analytical platforms for the study of affinity binding events and in the modification of nanostructures for biomedical applications. She is co-author of more than 150 publications, has written several book chapters, and holds six patents.

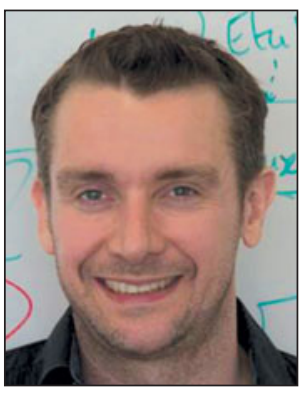

Oliver Williams

School of Physics and Astronomy, Cardiff University, UK; tel. +44 (0) 2920874978; and email williamso@cf.ac.uk.

Williams is a reader and Marie Curie Fellow at Cardiff School of Physics and Astronomy. He obtained his PhD degree from University College London. He worked at Argonne National Laboratory, the Institute for Materials Research, and the Fraunhofer Institute for Applied Solid State Physics prior to his current position. His research focuses on nanodiamond and superconductivity for quantum nanoelectromechanical systems and high-frequency filters.

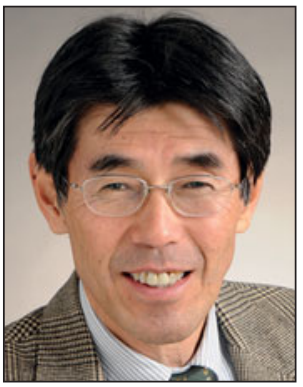

Satoshi Yamasak

National Institute of Advanced Industrial

Science and Technology, Japan;

email s-yamasaki@aist.go.jp.

Yamasaki is a principal research scientist at the Energy Technology Research Institute at the National Institute of Advanced Industrial Science and Technology with a concurrent position of Tsukuba University professor. He received his PhD degree in physics from Kyushu University in 1987. He is a research leader of the CREST project "Research for Ultra-Low Loss Power Devices" at JST and is a research fellow of the Japanese Applied Physics Society. He joined the Electrotechnical Laboratory in 1979 prior to his present position.

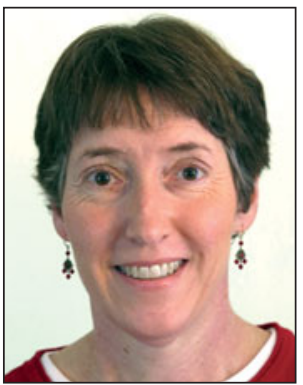

Joan Yater

Naval Research Laboratory, Washington, DC, USA; tel. 202-404-4494; and email joan.yater@nrl.navy.mil.

Yater is a research physicist at the Naval Research Laboratory (NRL) studying electron emission and cathode development for vacuum electronic device applications. She received her AB degree in physics from Cornell University and her PhD degree in physics from the University of Texas at Austin, followed by a National Research Council postdoctoral fellowship at NRL. She also researches diamond current amplification, photoemission, and thermionic emission.

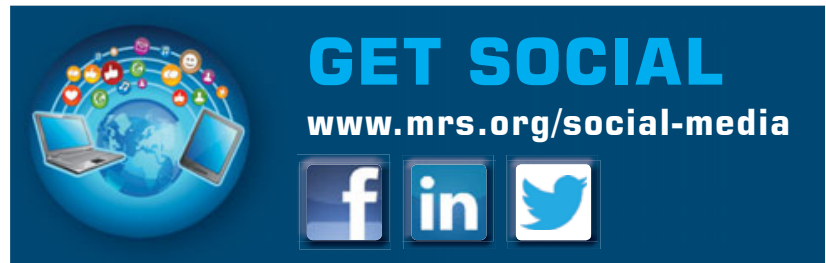

